\title{
ARTICLE \\ Neural activation to monetary reward is associated with amphetamine reward sensitivity
}

\author{
Natania A. Crane ${ }^{1}$, Stephanie M. Gorka ${ }^{1}$, Jessica Weafer ${ }^{2}$, Scott A. Langenecker ${ }^{1}$, Harriet de Wit ${ }^{2}$ and K. Luan Phan ${ }^{1,3}$
}

\begin{abstract}
One known risk factor for drug use and abuse is sensitivity to rewarding effects of drugs. It is not known whether this risk factor extends to sensitivity to non-drug rewards. In this study with healthy young adults, we examined the association between sensitivity to the subjective rewarding effects of amphetamine and a neural indicator of anticipation of monetary reward. We hypothesized that greater euphorigenic response to amphetamine would be associated with greater neural activation to anticipation of monetary reward (Win $>$ Loss). Healthy participants $(N=61)$ completed four laboratory sessions in which they received d-amphetamine $(20 \mathrm{mg})$ and placebo in alternating order, providing self-report measures of euphoria and stimulation at regular intervals. At a separate visit 1-3 weeks later, participants completed the guessing reward task (GRT) during fMRI in a drugfree state. Participants reporting greater euphoria after amphetamine also exhibited greater neural activation during monetary reward anticipation in mesolimbic reward regions, including the bilateral caudate and putamen. This is the first study to show a relationship between neural correlates of monetary reward and sensitivity to the subjective rewarding effects of amphetamine in humans. These findings support growing evidence that sensitivity to reward in general is a risk factor for drug use and abuse, and suggest that sensitivity of drug-induced euphoria may reflect a general sensitivity to rewards. This may be an index of vulnerability for drug use or abuse.
\end{abstract}

Neuropsychopharmacology (2018) 43:1738-1744; https://doi.org/10.1038/s41386-018-0042-8

\section{INTRODUCTION}

Excessive substance use and Substance Use Disorders (SUD) are a tremendous burden for the US economy, accounting for more than $\$ 740$ billion costs annually related to crime, lost work productivity, and health care [1]. In addition, individuals with SUD often suffer physical, psychological, and psychosocial costs [1]. Thus, it is crucial that we identify risk factors for SUD to inform prevention and intervention efforts. One risk factor that predicts future drug use is sensitivity to the rewarding effects of a single dose of a drug. Greater subjective response to drugs, especially drug-induced euphoria, is related to choosing to take the drug again, continued substance use, and development of SUD [2-11]. This relationship is referred to as reward or reinforcement: $[2,10]$. Indeed, drug-induced euphoria is a predictor of substance abuse and considered by the FDA to be an indicator of abuse liability of new medications [12]. However, little is known about the neural mechanisms underlying individual differences in sensitivity to drug reward, or nondrug rewards.

Most drugs of abuse activate brain reward circuitry and related neurotransmitter systems [13]. Positive subjective responses to single doses of stimulant drugs like amphetamine and methylphenidate are associated with greater dopamine release in the ventral striatum (VS), putamen, and caudate [14], as well as with dopamine cell activity, levels of D2 receptors, and level of D2/D3 receptor binding [15-19]. To our knowledge, however, no studies have yet examined how brain activity during non-drug reward is related to subjective response to a stimulant drug, a prototypic drug of abuse that elicits euphoria, among healthy, nondependent young adults.

Anticipation of non-drug rewards has been shown to activate the same reward regions as acute drug administration, indicating that neural response to drug-related rewards and non-drug rewards are similar [20]. This is likely due to dopamine neurotransmission in brain reward circuitry that is linked to motivation to seek and acquire rewards, both drug-related and non-drug rewards. Indeed, higher dopamine release has been associated with higher neural activation to non-drug, monetary reward among young adult male drinkers, both with or without a family history of Alcohol Use Disorder [21]. It has also been noted that individuals differ in the degree of neural activation to anticipation of non-drug rewards. For example, individuals with high-reward sensitivity, as measured by self-report personality measures, show increased neural activation in brain reward circuitry, particularly in the VS and the orbitofrontal cortex (OFC), during non-drug reward anticipation compared to individuals with low-reward sensitivity $[22,23]$. This individual variability in brain reward function may influence drug use via drug craving, biasing toward drug cues, and motivation to seek the drug [24, 25]. Yet, no studies have directly examined whether brain reward activation during non-drug reward (in the absence of drug) is related to subjective response to drug, a key risk factor for SUD,

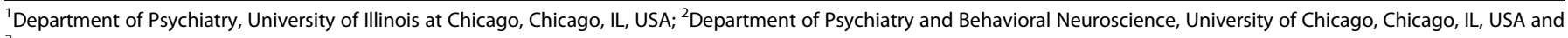
${ }^{3}$ Mental Health Service Line, Jesse Brown VA Medical Center, Chicago, IL, USA

Correspondence: Natania A. Crane (ncrane3@uic.edu)

These authors contributed equally: Harriet de Wit, K. Luan Phan.
}

Received: 8 November 2017 Revised: 1 February 2018 Accepted: 27 February 2018

Published online: 14 March 2018 
Table 1. Participant characteristics

\begin{tabular}{ll}
\hline & $\begin{array}{l}\text { Healthy young adults } \\
(n=61)\end{array}$ \\
\hline Age & $24.57(2.62)$ \\
Gender (\% female) & $51 \%$ \\
Ethnicity (\% hispanic) & $15 \%$ \\
Race & \\
$\%$ Caucasian & $65 \%$ \\
$\%>1$ Race & $10 \%$ \\
$\%$ African-American & $15 \%$ \\
$\%$ Asian & $8 \%$ \\
$\%$ American Indian/Alaskan Native & $2 \%$ \\
Current substance use & \\
Alcohol (drinks/week) & $2.53(1.26)$ \\
Cigarettes (per day) & $0.80(1.66) ; n=13$ \\
Caffeine (cups/day) & $1.49(0.95) ; n=56$ \\
Marijuana (times/month) & $5.53(7.89) ; n=17$ \\
Lifetime substance use (\% ever used) & \\
Marijuana & $70 \%$ \\
Hallucinogens & $23 \%$ \\
Stimulants & $23 \%$ \\
Opiates & $15 \%$ \\
MDMA & $15 \%$ \\
Sedatives & $7 \%$ \\
Subjective response to drug & \\
ARCl-MBG peak change difference score & $4.75(4.58)$ \\
\hline Alvalues are means and standard devations &
\end{tabular}

All values are means and standard deviations unless otherwise noted.

among non-dependent young adults before drug use problems have developed.

Taken together, individuals who find drugs to be subjectively rewarding are at risk for SUD and there is reason to suggest that this reward sensitivity extends beyond just drugs of abuse and is a broader individual difference factor. Thus, individuals who are sensitive to the rewarding effects of drug may also respond more strongly to non-drug rewards, perhaps because of individual differences in brain reward function. As such, the goal of the current study was to examine the association between neural activity during anticipation of monetary reward and sensitivity to the subjective rewarding effects of oral d-amphetamine in healthy young adults. Neural responses during monetary reward anticipation were assessed using fMRI while subjects performed the guessing reward task (GRT), which has been shown to activate brain reward circuitry [26]. Euphorigenic effects of d-amphetamine were assessed with standardized questionnaires during four double-blind challenge doses of amphetamine or placebo. We hypothesized that greater neural activation to monetary reward anticipation would be associated with greater subjective response to amphetamine.

\section{MATERIALS AND METHODS}

Design

Participants completed four drug challenge sessions to assess their subjective responses to $\mathrm{d}$-amphetamine $(20 \mathrm{mg}$ oral) or placebo, and a single-scanning session without drug administration. During the drug challenge sessions participants received $d$ amphetamine and placebo twice, in alternating order, with order of the first drug administered (d-amphetamine or placebo) randomly assigned. During the fMRI scanning visit 1-3 weeks later they completed the GRT, in a drug-free state. The Institutional Review Board at University of Chicago (UC) and University of Illinois of Chicago (UIC) approved the study and written informed consent was obtained.

\section{Participants}

Participants were right-handed, healthy young adults aged 21-35 who reported occasional nonmedical substance use and were recruited from nearby college campuses and surrounding communities through online and printed advertisements. Of the 88 individuals initially enrolled, eight participants dropped out of the study before completing all laboratory visits, 10 participants were missing imaging data for the GRT task (they did not complete the GRT task in the scanner), and nine participants were excluded from analyses for significant movement (as described below). This left a final sample size of 61 (Table 1). Inclusion criteria included body mass index between 19 and 26, at least a high school education, and English fluency. Exclusion criteria were current or past year DSM-IV diagnosis [27], lifetime history of SUD, serious medical conditions, night shift work, positive urine drug screen on any session and contraindication for fMRI. Participants were excluded if they had a past year DSM-IV diagnosis, lifetime history of substance dependence or ADHD, a serious medical condition, smoked $>5$ cigarettes per day, used medications daily (other than birth control), or if they were pregnant, lactating, or planning to become pregnant in the next 3 months. Females not on hormonal contraception completed drug administration sessions in the follicular phase of their menstrual cycle [28].

\section{Measures}

Addiction Research Center Inventory (ARCl; [29]). To assess subjective response to d-amphetamine, participants completed the ARCI [30]. The Morphine-Benzedrine group (MBG, euphoric effects) scale and the Amphetamine-like ( $A$, stimulant effects) scale were used, as these have been shown to represent the positive, rewarding effects of amphetamine (e.g., refs. [10, 31]). The peak change difference scores for ARCI-MBG and ARCI-A (average $\mathrm{d}$-amphetamine peak change score minus average placebo peak change score; [32]) were used for analyses.

Guessing Reward Task (GRT). Participants completed an adaptation of a GRT [25] that is designed to capture neural activation to reward anticipation. The task, shown in Fig. 1, consisted of 60 trials (15 win, 15 loss, 15 no-win and 15 no-loss). Each trial included a

\section{Guessing Reward Task (GRT)}

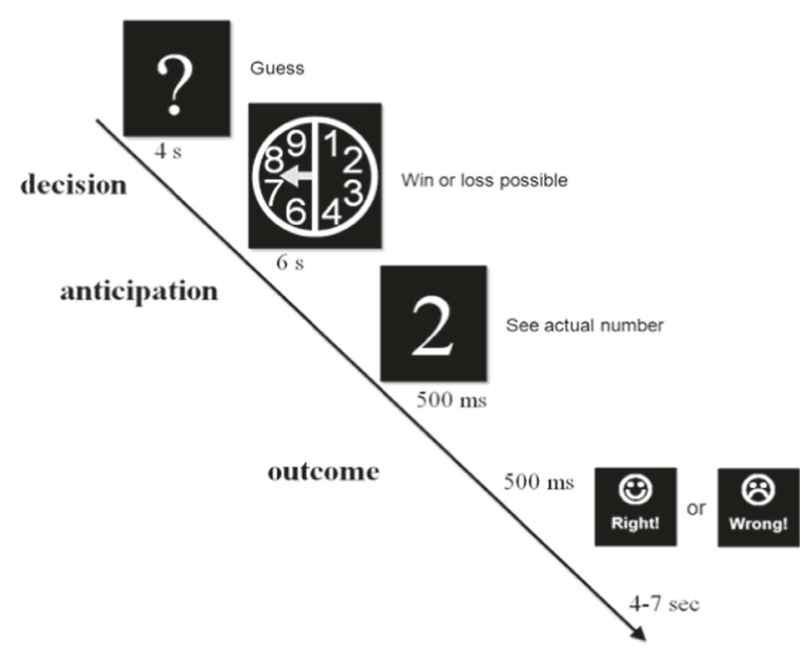

Fig. 1 Guessing reward task 
decision, anticipation, and outcome period, and trials were separated by an inter-trial interval from 4 to $7 \mathrm{~s}$. During the decision period, participants were presented with a question mark $(4 \mathrm{~s})$ and pressed a button to guess whether an upcoming computer-selected number would be greater than or less than 5 . For the anticipation phase, participants viewed a circle with the numbers 1-9 and a yellow arrow indicating the range of the actual number, which informed participants whether their choice was correct or incorrect (presented for $6 \mathrm{~s}$ ). Participants were informed that they might win $\$ 1$ or no money for a correct response, but they might lose 50 cents or no money for an incorrect response. Immediately after they made a response for a trial, participants were told whether their choice was correct (win trial) or incorrect (loss trial) (as indicated by the yellow arrow described above), during the "anticipation phase" of the trial. Then, during the "outcome phase", they were told how much they had won or lost. Therefore, although participants knew whether it was a win or loss trial during the anticipation phase, they did not know whether they would receive $\$ 1$ or no money for a win trial and whether they would lose 50 cents or no money for a loss trial until the outcome phase of the trial. During the outcome phase, participants were presented with the "actual" number for $500 \mathrm{~ms}$ and received feedback for $500 \mathrm{~ms}$ in the form of a happy face for wins, sad face for losses, and neutral face for breaking even, presented together with information about how much money they won or lost for that trial. Participants also saw their total earnings every 20 trials $(\$ 2.50, \$ 4.50, \$ 7.50)$. They were told they had a chance of winning between $\$ 0$ and $\$ 15.00$ at the end of the task depending on their performance. However, unbeknownst to participants, the task was rigged, so their behavior did not affect actual outcomes, and therefore was not analyzed or reported. Each participant received $\$ 10$. The contrast of interest in the imaging data was Win > Loss during the anticipation phase. This contrast between Win and Loss has been shown to be more a sensitive indicator of neural activation during reward processing than examining Win or Loss individually [33-35] and striatal activation has been shown to increase during Win events and decrease during Loss events [36]. It is important to note that the task does not provide a valid measure of 'neutral' anticipation, as participants do not know that the trial is a neutral trial until the outcome phase when they are presented with their monetary outcome for that trial.

\section{Study procedure}

Participants completed an initial screening and orientation visit during which they provided informed consent and were familiarized with laboratory procedures and study protocol. Participants completed drug administration sessions at UC and then attended a separate fMRI visit at UIC 1-3 weeks later. Participants were asked to abstain from drugs, including alcohol, for $24 \mathrm{~h}$ prior to each visit, which was verified by self-report, breath alcohol, and urine screens.

During the four drug administration sessions participants received d-amphetamine $(20 \mathrm{mg})$ and placebo in alternating order. D-amphetamine and placebo were each administered twice to minimize the influence of day-to-day variability [37]. Sessions took place from 09:00 to 13:00 h in comfortable, livingroom-like rooms, and were separated by at least $48 \mathrm{~h}$. Participants were tested individually. Participants were instructed not to eat after midnight before each session and were given a light snack upon arrival to the lab. To minimize drug expectancies they were told they could receive one of the following: stimulant, sedative, or placebo. On each session subjects first completed pre-drug $A R C I$, and had blood pressure and heart rate measured. At 09:20 h, damphetamine ( $20 \mathrm{mg}$ oral; Desoxyn tablets with dextrose filler in size 00 opaque capsules) or placebo (dextrose only) was administered under double-blind conditions. Participants completed the $\mathrm{ARCl}$ every $30 \mathrm{~min}$ following capsule administration.
Sessions ended at 13:00 $\mathrm{h}$, after confirmation that blood pressure and heart rate had returned to baseline.

The fMRI session took place on a separate day, at least 1 week after the last drug administration session. Participants were tested for recent drug use and screened for MRI safety before completing the GRT during the scan. After completing all sessions, participants were debriefed and compensated for their participation.

\section{fMRI data acquisition}

Functional MRI data were collected using a $3 \mathrm{~T}$ GE magnetic resonance scanner at the UIC Center for Magnetic Resonance Research. Functional images were acquired using a gradient-echo echo-planar images ( $2 \mathrm{~s}$ TR, $25 \mathrm{~ms} \mathrm{TE}, 82^{\circ}$ flip, $64 \times 64$ matrix, 200 $\mathrm{mm}$ FOV, $3 \mathrm{~mm}$ slice thickness, $0 \mathrm{~mm}$ gap, with 44 axial slices).

\section{fMRI data analyses}

Imaging data were inspected and individuals with $>2 \mathrm{~mm}$ displacement in any direction were not included in the analysis. The remaining subjects met criteria for high quality and scan stability. Preprocessing of fMRI data were conducted using Statistical Parametric Mapping software (SPM12, Wellcome Department of Imaging Neuro-Science, London, UK). Images were spatially realigned, slice-time corrected, warped to Montreal Neurological Institute (MNI) space using the participant's mean functional image, resampled to $2 \mathrm{~mm}^{3}$ voxels, and smoothed (4 $\mathrm{mm}^{3}$ kernel). The general linear model was applied to the time series, convolved with the canonical hemodynamic response function and with a 128-s high-pass filter. Condition effects were modeled with event-related regressors representing the occurrence of anticipation for Win or Loss. Effects were estimated at each voxel, and for each subject. Individual contrast maps for Win trials (>Loss trials) were created for each person. Individual motion parameter files were included in the first levels models as regressors-of-no-interest.

To test our hypotheses, contrast maps for Win > Loss anticipation were entered into second-level, one-sample $t$-test in SPM. This approach allowed for an examination of the main effects of the task, independent of drug-induced euphoria ratings. To confirm that the task successfully activated reward-related regions during Win > Loss trials, we examined task activation across all subjects. Next, individual drug-induced $\mathrm{ARCl}$ scores were entered as a regressor of interest for the Win > Loss anticipation second-level model. As our hypothesis was specific to reward-related regions, neural activity from these models were considered significant if it exceeded correction of multiple comparisons across a functional reward mask downloaded from Neurosynth [38] as determined via simulation using the $3 d$ ClustSim utility $(10,000$ iterations; updated and 'bug-free' on December 2015; [https://afni.nimh.nih.gov/pub/ dist/doc/program_help/3dClustSim.html]; [39]). A reverse inference mask was downloaded from Neurosynth in July 2017 from a meta-analyses of 532 studies with 100 terms relating to "reward" and displaying regions that are reported more often in studies that load highly on "reward" (http://neurosynth.org/analyses/ topics/v4-topics-100/99). Significance at corrected $a<0.05$ and a voxel threshold of $p<0.005$ yielded a minimum cluster size of at least 94 contiguous voxels (volume $=752 \mathrm{~mm}^{3}$ ). In order to show the distribution of activation data in a scatterplot, we extracted parameter estimates $/ \beta$-weights from a $10 \mathrm{~mm}$ radius sphere surrounding the peak activation.

\section{RESULTS}

Task activation during reward

Reward anticipation relative to loss (Win > Loss) significantly activated a large contiguous cluster of frontal and mesolimbic reward regions, including bilateral nucleus accumbens, caudate, and putamen (peak MNI $[-6,16,-10], k=3376$ voxels, $Z=7.30$, 


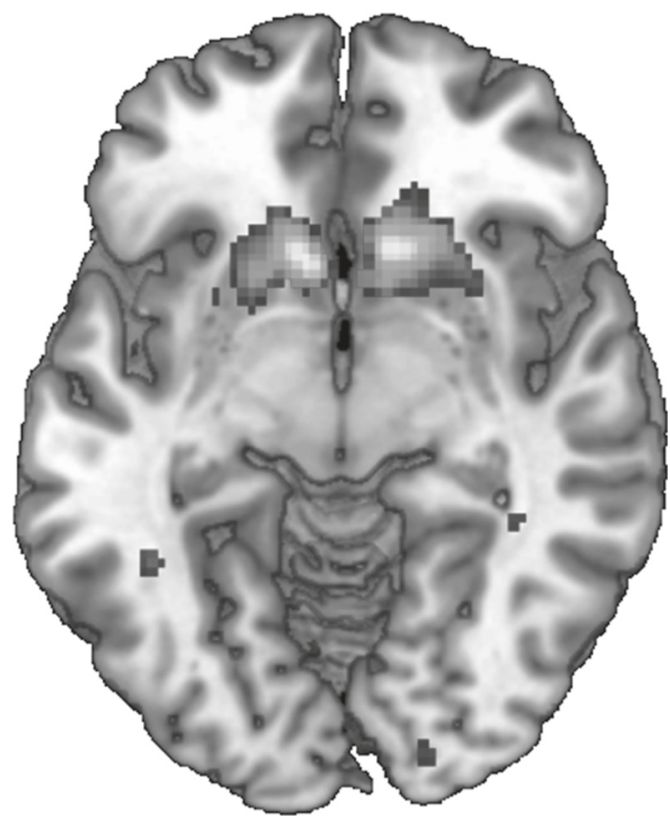

$t$-value

3.8

Fig. 2 Task activation during reward within the neurosynth reward mask. Task activation within the neurosynth reward mask $(p<0.05$, corrected) for all subjects to reward anticipation (Win > Loss trials)

Table 2. Task activation during reward within the neurosynth reward mask

\begin{tabular}{ll}
\hline Lobe & $\begin{array}{l}\text { MNI } \\
\text { coordinates }\end{array}$ \\
& $\frac{\text { Z-score }}{x y z}$ Voxels \\
& (k)
\end{tabular}

\section{Frontal/Subcortical}

$\begin{array}{lllllll}\text { Contiguous cluster extending from } & -6 & 16 & -10 & 7.30 & 3,376\end{array}$ the bilateral insula and inferior frontal gyrus to the caudate

$L$ inferior frontal gyrus $\begin{array}{llll}-42 & 2 & 36 & 4.14\end{array}$

261

Anterior cingulate extending to the

$\begin{array}{llll}-4 & 54 & -2 & 4.12\end{array}$

orbitofrontal cortex

$\mathrm{R}$ inferior frontal gyrus

$\begin{array}{llll}44 & 12 & 24 & 3.90\end{array}$

Reporting of all significant peak voxels within the neurosynth mask at $p<.05$ corrected with a cluster size of $\geq 94$ contiguous voxels $L$ left, $R$ right, $M N I$ Montreal Neurologic Institute

$p<.05$, corrected). Mesolimbic activation during reward is illustrated in Fig. 2. Significant peak clusters within the Neurosynth mask are shown in Table 2.

\section{Subjective effects of amphetamine}

As previously reported in this dataset [40, 41], d-amphetamine significantly increased ratings of euphoria [mean amphetamine score $=4.6 \quad(\mathrm{sd}=4.7)$, mean placebo score $=-0.1 \quad(\mathrm{sd}=2.3)$; paired samples t-test: $t(60)=8.1, p<0.001$ ] and stimulation [mean amphetamine score $=3.5(\mathrm{sd}=2.9)$, mean placebo score $=-0.1$ $(\mathrm{sd}=1.6)$, paired samples $t$-test: $t(60)=9.2, p<0.001]$, relative to placebo. Individuals differed in their responses to d-amphetamine, with peak difference scores (d-amphetamine minus placebo) ranging from -5.5 to 17 for euphoria and -4 to 12.5 for stimulation.

Association between neural activation during reward and subjective response to amphetamine

More BOLD activation during Win > Loss anticipation was associated with greater drug-induced euphoria (peak change difference ARCI-MBG score) in a large contiguous cluster that included the bilateral putamen and caudate (right: MNI peak $[16,6,6], k=$ 266 voxels, $Z=3.62, p<.05$, corrected; left: MNI peak [-20, 12, 2], $k=203$ voxels, $Z=3.45, p<.05$, corrected) (Fig. 3) $)^{1,2}$. Druginduced stimulation (peak change difference ARCI-A score) was not associated with BOLD activation within the mask during Win $>$ Loss anticipation.

\section{Exploratory analyses}

To better understand whether Win events or Loss events contributed to the association between Win > Loss anticipation and drug-induced euphoria, we examined if Win > Fixation and Loss > Fixation were separately related to drug-induced euphoria. For these exploratory analyses, individual drug-induced ARCI-MBG scores were entered as a regressor of interest for the Win > Fixation anticipation model and for the Loss > Fixation anticipation model. Neither model was significant, indicating that the original findings were due to the relative difference between Wins vs. Losses.

To determine whether any of the subjects' responses were related to prior drug use, we examined the relationships among drug-induced euphoria, parameter estimates/ $\beta$-weights of BOLD peak activation during Win $>$ Loss anticipation, and current and lifetime substance use measures. Neither drug-induced euphoria nor BOLD peak activation during Win > Loss anticipation were related to current (past month) alcohol, cigarette, caffeine, or marijuana use (Spearman Rho correlations $p$-values $>.05$ ), or to whether participants had ever (vs never) used marijuana, hallucinogens, stimulants, opiates, MDMA, or sedatives in their lifetime ( $t$-test $p$-values $>.05)$.

\section{DISCUSSION}

One predictor of risk for drug use and abuse is sensitivity to the rewarding effects of drugs, and perhaps also sensitivity to rewards in general. Here, we studied brain responses to a monetary reward in relation to sensitivity to the subjective rewarding effects of amphetamine in healthy young adults. We found novel evidence that greater activation in mesolimbic reward regions during anticipation of monetary reward in a drug-free state was associated with greater euphoria after d-amphetamine administration. Specifically, individuals who reported greater subjective euphoria after amphetamine during the behavioral phase of the study exhibited greater neural activation during reward anticipation in the bilateral caudate and right putamen. Notably, the relative difference between Win and Loss events, rather than Win events or Loss events separately (although see limitations of these analyses below), was significantly related to subjective drug response. This is the first study to show a relationship between neural correlates of monetary reward (in the absence of drug) and sensitivity to the subjective rewarding effects of a stimulant drug in humans.

The finding that activation in the caudate and putamen was related to subjective drug reward is consistent with what is known about both brain reward circuitry and risk factors involved in drug use and abuse. The striatum, including the caudate and the putamen, are involved in many aspects of reward evaluation and incentive-based learning (see ref. [20]), and the striatum has been linked to both the initiation and maintenance of substance use behaviors [13]. A recent study found that greater dopamine release was associated with both an earlier age of first 


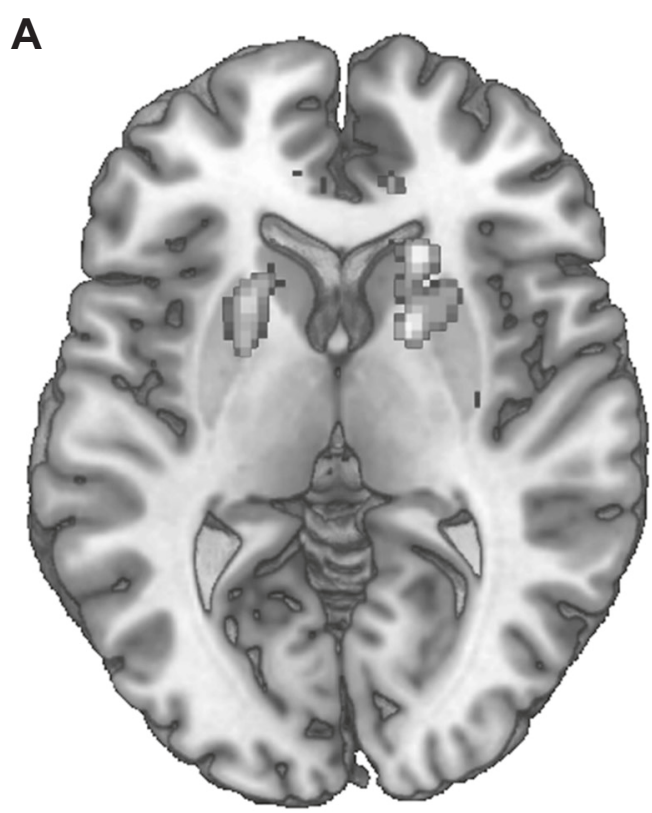

$t$-value

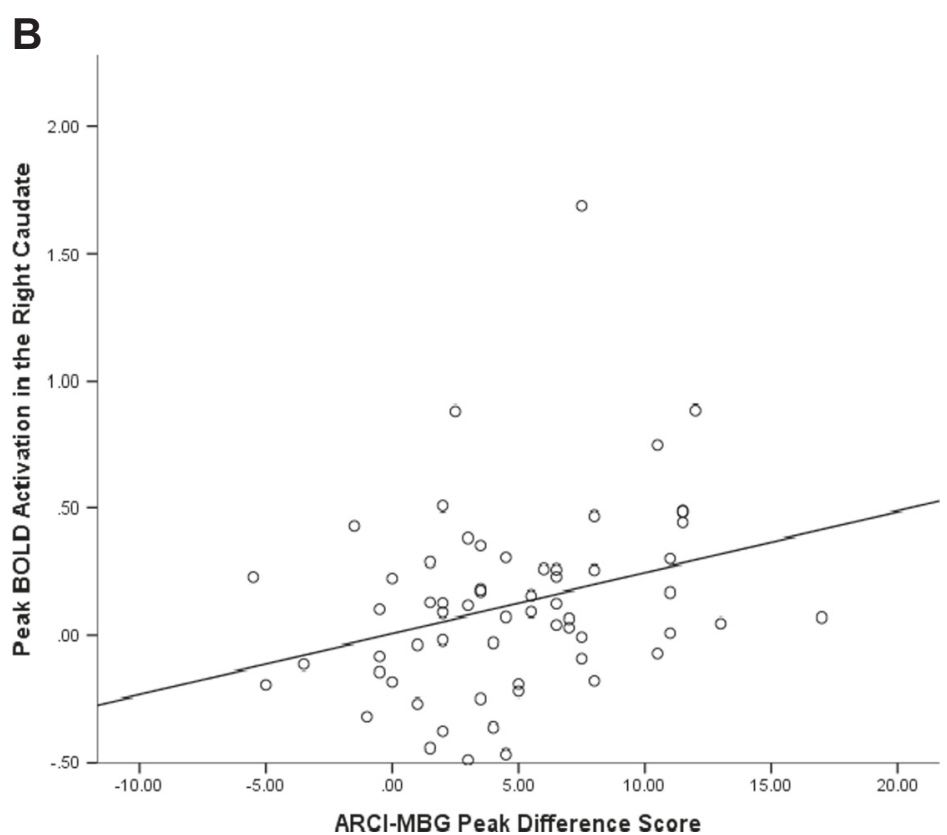

Fig. 3 Greater euphoria response to amphetamine is associated with greater activation during reward anticipation. a shows significant ( $p<$ 0.05 , corrected) peak activation observed in the bilateral caudate and putamen. $\mathbf{b}$ illustrates the relationship between greater drug-induced euphoria (ARCI-MBG) and mesolimbic activation in the right caudate (MNI peak activation $[16,6,6])$ in order to show the distribution of the data.

drunkenness and greater neural activation to monetary reward in high-risk youth (Weiland et al., 2017), supporting the notion that more striatal activation to non-drug, monetary reward may be a neural profile for vulnerability for drug use problems. Similarly, subjective response to drug reward has been shown to be an important risk factor for several indicators of substance use problems, including choosing to take the drug again, progression of substance use, and development of SUD [211]. Therefore, the current study establishes an important link between activation in the striatum, a known neural mechanism involved in both reward and substance abuse, and euphoric response to drug reward among healthy, non-dependent young adults.

In addition, the current study bridges two separate, but related, literatures on neural response to non-drug reward and neural response to acute drug administration. It extends observations that individuals who score high on personality measures of reward sensitivity show increased neural activation in brain reward circuitry during non-drug reward [21, 22], by showing that greater subjective reward to a drug challenge is related to greater neural activation in the striatum during non-drug reward. Further, previous studies showed that acute amphetamine and methylphenidate increased dopamine release in the striatum [14-19], while our study showed that greater subjective rewarding effects of amphetamine are also related to increased activation in the striatum during non-drug reward (when individuals are not acutely intoxicated). Taken together, these findings demonstrate a similar pattern of increased activation in the striatum in response to drug and non-drug reward.

In this sample of relatively light drug users, previous substance use was not related to drug-induced euphoria or to BOLD peak activation during Win $>$ Loss anticipation. This suggests that the relationships reflect stable, individual differences that are not the result of prior drug exposure. The findings suggest that a proportion of relatively light drug users may be at elevated risk for future onset of drug use problems. These relationships may be even stronger among individuals who were not tested here because they had already developed problems. Alternatively, these individuals may indeed be at risk, but were protected by other factors that prevent the development of excessive drug use. Future prospective studies will be needed to determine whether either acute drug responses or neural responses to reward predict development of problem use.

These findings have important implications. Greater neural activation to non-drug, monetary reward may represent a risk factor for SUD among young adults. Greater neural activation during reward is linked to greater hedonia and subjective pleasure from drug rewards and this, in turn could lead to more drugseeking. Therefore, we may be able to use neural activation to monetary reward to detect this broader trait early on, before substance use initiation or progression. This may be particularly useful in adolescent populations, in whom it is not possible to study responses to drugs.

Despite the strengths of the study (e.g., a relatively large sample size and separate measures of drug and non-drug reward), the study also had limitations. The participants were healthy young adults, and it is not clear that the findings would be generalizable to individuals who are at high risk, such as those with a family history of drug use. Another limitation was that the behavioral drug sessions always preceded the $\mathrm{fMRI}$, raising the possibility that exposure to the drug changed brain responses to monetary reward. However, it is unlikely that single low doses of $d$ amphetamine influenced brain activity 1-3 weeks later. Further, we tested only one dose of amphetamine, and full dose-response data with amphetamine would provide a more complete picture of the pharmacological profile. There were also limitations related to the task used during the imaging session. Because the GRT task does not have a neutral anticipation condition in which subjects expect no money to be won or lost, there is no true control condition to compare against Win and Loss anticipation events. 
Our attempt to examine the separate effects of Win and Loss events using Fixation is not a comparable "control" condition. It will important for future studies to determine the respective contributions of Win and Loss anticipation using a 'neutral' condition. The GRT also did not allow us to examine neural activation during reward receipt, which would be an interesting and relevant construct. Our study did not assess family history of drug use, which might influence activation to reward (see ref. [41]). Although, we typically detect a low prevalence of family history of alcoholism in drug challenge studies in our laboratory, it would be of interest to examine family history of SUD in relation to drug-induced euphoria and neural reward activation. Finally, our study is cross-sectional and we did not measure substance use over time. Future and ongoing studies are needed to replicate the current study in longitudinal samples.

Our study provides novel evidence of a relationship between neural correlates of monetary reward in the absence of drug and sensitivity to the subjective rewarding effects of amphetamine in humans. Specifically, we found that greater activation in mesolimbic reward regions, including the bilateral caudate and putamen, during non-drug reward anticipation was associated with more drug-induced euphoria. These findings extend our understanding of the neural correlates of a well-known risk factor for SUD, sensitivity of drug-induced euphoria, and provide evidence that individual differences to non-drug reward and subjective drug reward are linked and could represent a profile of vulnerability for SUD.

\section{FUNDING}

This publication was funded by the National Institute on Drug Abuse (NIDA) (R01DA002812, Pls: H.d.W. and K.L.P.). S.M.G. was supported by the National Institute on Alcohol Abuse and Alcoholism (NIAAA) (K23AA025111, PI: S.M.G.) and J.W. was supported by NIAAA (K01AA024519, PI: J.W.). Its contents are solely the responsibility of the authors and do not necessarily represent the official views of NIDA, NIAAA, or the National Institutes of Health.

\section{ADDITIONAL INFORMATION}

Competing interests: H.d.W. received consulting fees from Jazz Pharmaceuticals, research support in the form of a GRAND award from Pfizer, support for a research study from Insys Therapeutics, and a donation of a research drug from Indivior. None of these were related to the research reported here. All the other authors declare no competing interests.

\section{REFERENCES}

1. NIDA (2017). Trends \& Statistics. Retrieved from: https://www.drugabuse.gov/ related-topics/trends-statistics.

2. Haertzen CA, Kocher TR, Miyasato K. Reinforcements from the first drug experience can predict later drug habits and/or addiction: results with coffee, cigarettes, alcohol, barbiturates, minor and major tranquilizers, stimulants, marijuana, hallucinogens, heroin, opiates and cocaine. Drug Alcohol Depend. 1983;11:147-65.

3. King AC, McNamara PJ, Hasin DS, Cao D. Alcohol challenge responses predict future alcohol use disorder symptoms: a 6-year prospective study. Biol Psychiatry. 2014;75:798-806.

4. Kollins SH, MacDonald EK, Rush CR. Assessing the abuse potential of methylphenidate in nonhuman and human subjects: a review. Pharmacol Biochem Behav. 2001;63:611-27.

5. Lambert NM, McLeod M, Schenk S. Subjective responses to initial experience with cocaine: an exploration of the incentive-sensitization theory of drug abuse. Addiction. 2006;101:713-25.

6. Quinn PD, Fromme K. Subjective response to alcohol challenge: a quantitative review. Alcohol Clin Exp Res. 2011;35:1759-70.

7. Rush CR, Essman WD, Simpson CA, Baker RW. Reinforcing and subject-rated effects of methylphenidate and d-amphetamine in non-drug-abusing humans. J Clin Psychopharmacol. 2001;21:273-86.

8. Volkow ND, Wang GJ, Fowler JS, Logan J, Gatley SJ, Wong C, Pappas NR. Reinforcing effects of psychostimulants in humans are associated with increases in brain dopamine and occupancy of $\mathrm{D}(2)$ receptors. J Pharmacol Exp Ther. 1999;291:409-15.

9. Davidson ES, Finch JF, Schenk S. Variability in subjective responses to cocaine: initial experiences of college students. Addict Behav. 1993;18: 445-53.

10. de Wit $\mathrm{H}$, Phillips TJ. Do initial responses to drugs predict future use or abuse? Neurosci Biobehav Rev. 2012;36:1565-76.

11. de Wit $\mathrm{H}$, Uhlenhuth $\mathrm{EH}$, Johanson $\mathrm{CE}$. Individual differences in the reinforcing and subjective effects of amphetamine and diazepam. Drug Alcohol Depend. 1986;16:341-60.

12. Jasinski DR. History of abuse liability testing in humans. $\mathrm{Br} J$ Addict. 1991;86:1559-62.

13. Koob GF, Volkow ND. Neurobiology of addiction: a neurocircuitry analysis. Lancet Psychiatry. 2016;3:760-73.

14. Oswald LM, Wong DF, McCaul M, Zhou Y, Kuwabara H, Choi L, et al. Relationships among ventral striatal dopamine release, cortisol secretion, and subjective responses to amphetamine. Neuropsychopharmacology. 2005;30: 821-32.

15. Abi-Dargham A, Kegeles LS, Martinez D, Innis RB, Laruelle M. Dopamine mediation of positive reinforcing effects of amphetamine in stimulant naive healthy volunteers: results from a large cohort. Eur Neuropsychopharmacol. 2003;13:459-68.

16. Leyton M, Boileau I, Benkelfat C, Diksic M, Baker G, Dagher A. Amphetamineinduced increases in extracellular dopamine, drug wanting, and novelty seeking: a PET/[11C]raclopride study in healthy men. Neuropsychopharmacology. 2002;27:1027-35.

17. Martinez D, Slifstein M, Broft A, Mawlawi O, Hwang DR, Huang $Y$, et al. Imaging human mesolimbic dopamine transmission with positron emission tomography. Part II: amphetamine-induced dopamine release in the functional subdivisions of the striatum. J Cereb Blood Flow Metab. 2003:23:285-300.

18. Volkow ND, Fowler JS, Wang GJ, Ding YS, Gatley SJ. Role of dopamine in the therapeutic and reinforcing effects of methylphenidate in humans: results from imaging studies. Eur Neuropsychopharmacol. 2002;12:557-66.

19. Drevets WC, Gautier C, Price JC, Kupfer DJ, Kinahan PE, Grace AA, et al. Amphetamine-induced dopamine release in human ventral striatum correlates with euphoria. Biol Psychiatry. 2001;49:81-96.

20. Haber SN, Knutson B. The reward circuit: linking primate anatomy and human imaging. Neuropsychopharmacology. 2010;35:4-26.

21. Weiland BJ, Zucker RA, Zubieta JK, Heitzeg MM (2017). Striatal dopaminergic reward response relates to age of first drunkenness and feedback response in atrisk youth. Addict Biol 22:502-512.

22. Hahn T, Dresler T, Ehlis AC, Plichta MM, Heinzel S, Polak T, et al. Neural response to reward anticipation is modulated by Gray's impulsivity. Neuroimage. 2009;46:1148-53.

23. Cohen MX, Young J, Baek JM, Kessler C, Ranganath C. Individual differences in extraversion and dopamine genetics predict neural reward responses. Brain Res Cogn Brain Res. 2005;25:851-61.

24. Leyton M, Vezina P. Striatal ups and downs: their roles in vulnerability to addictions in humans. Neurosci Biobehav Rev. 2013;37:1999-2014.

25. Saunders BT, Robinson TE. Individual variation in resisting temptation: implications for addiction. Neurosci Biobehav Rev. 2013;37:1955-75.

26. Forbes EE, Hariri AR, Martin SL, Silk JS, Moyles DL, Fisher PM, et al. Altered striatal activation predicting real-world positive affect in adolescent major depressive disorder. Am J Psychiatry. 2009;166:64-73.

27. First $M$, Spitzer R, Gibbon M, Williams J. Structured Clinical Interview for DSM-IV ${ }^{\circledR}$ Axis I Disorders (SCID-I), Clinician Version, Administration Booklet (2002).

28. White $T L$, Justice $A J$, de Wit $H$. Differential subjective effects of D-amphetamine by gender, hormone levels and menstrual cycle phase. Pharmacol Biochem Behav. 2002;73:729-41.

29. Martin WR, Sloan JW, Sapira JD, Jasinski DR. Physiologic, subjective, and behavioral effects of amphetamine, methamphetamine, ephedrine, phenmetrazine, and methylphenidate in man. Clin Pharmacol Ther. 1971;12:245-58.

30. Fischman MW, Foltin RW. Utility of subjective-effects measurements in assessing abuse liability of drugs in humans. Br J Addict. 1991;86:1563-70.

31. Mayo LM, de Wit $\mathrm{H}$. Acquisition of responses to a methamphetamine-associated cue in healthy humans: self-report, behavioral, and psychophysiological measures. Neuropsychopharmacology. 2015;40:1734-41.

32. Forbes EE, Rodriguez EE, Musselman S, Narendran R. Prefrontal response and frontostriatal functional connectivity to monetary reward in abstinent alcoholdependent young adults. PLoS ONE. 2014;9:e94640.

33. Foti D, Weinberg A, Dien J, Hajcak G. Event-related potential activity in the basal ganglia differentiates rewards from nonrewards: temporospatial principal 
1744

components analysis and source localization of the feedback negativity. Hum Brain Mapp. 2011;32:2207-16.

34. Carlson JM, Foti D, Mujica-Parodi LR, Harmon-Jones E, Hajcak G. Ventral striatal and medial prefrontal BOLD activation is correlated with reward-related electrocortical activity: a combined ERP and fMRI study. Neuroimage. 2011;57:1608-16.

35. Proudfit GH. The reward positivity: from basic research on reward to a biomarker for depression. Psychophysiology. 2015;52:449-59.

36. Delgado MR, Nystrom LE, Fissell C, Noll DC, Fiez JA. Tracking the hemodynamic responses to reward and punishment in the striatum. J Neurophysiol. 2000;84:3072-7.

37. Rhodes JD, Hawk LW Jr. Smoke and mirrors: the overnight abstinence paradigm as an index of disrupted cognitive function. Psychopharmacology. 2016;233: 1395-404
38. Yarkoni T, Poldrack RA, Nichols TE, Van Essen DC, Wager TD. Large-scale automated synthesis of human functional neuroimaging data. Nat Methods. 2011;8:665-70

39. Cox RW. AFNI: software for analysis and visualization of functional magnetic resonance neuroimages. Comput Biomed Res. 1996;29:162-73.

40. Weafer J, Gorka SM, Hedeker D, Dzemidzic M, Kareken DA, Phan KL, et al. Associations between behavioral and neural correlates of inhibitory control and amphetamine reward sensitivity. Neuropsychopharmacology. 2017a;42: 1905-13.

41. Weafer J, Lyon N, Hedeker D, de Wit H. Sweet taste liking is associated with subjective response to amphetamine in women but not men. Psychopharmacology. 2017b;234:3185-94. 